30 days. European data are sparse. We aimed to define the readmission rate in Scotland and identify reasons and predictors for readmission.

Methods Patients undergoing primary transplant with cirrhosis between January 2009 and December 2018 were included $(n=639)$. Data were collected on patient and disease demographics and blood results at transplant and discharge. Differences between those readmitted and not readmitted at 30 days were assessed using Chi-squared or Mann-Whitney U tests. Survival was assessed using Kaplan-Meier analysis. Cox proportional hazards were used to predict readmissions.

Results Patients were predominantly male $(n=410 ; 64.2 \%)$ with a median age of 58.9 years (IQR 51.8-64.1) at transplant. The commonest aetiologies were alcohol-related liver disease $(n=226 ; 35.4 \%)$, chronic viral hepatitis $(n=111$; $17.4 \%)$ and non-alcoholic fatty liver disease $(n=104 ; 16.4 \%)$. 208 patients $(32.6 \%)$ had a hepatocellular carcinoma. Patients had a median UKELD of 55 (52-59) and a median length of stay of 13 days (10-18). One year mortality was $4.1 \%$ $(n=26)$.

Readmission rates were: 30 days, 19.4\% ( $n=124)$; 90 days, $30.6 \%(n=194) ; 1$ year, 46.9\% $(n=300)$. Demographics and blood results were similar between those readmitted at 30 days and those not, although significant differences were haemoglobin $(\mathrm{g} / \mathrm{dL})$ at transplant (readmitted vs not readmitted) (105 vs. $111 ; \mathrm{p}=0.02)$, urea $(\mathrm{mmol} / \mathrm{L})$ at discharge $(7.5$ vs $6.3 ; \mathrm{p}=0.009)$, and creatinine $(\mathrm{mmol} / \mathrm{L})$ at discharge (80 vs 73; $\mathrm{p}=0.007)$.

Readmission within 30 days post LT conferred a significantly higher 1-year mortality $(10 \quad(8.1 \%)$ vs. $16 \quad(3.1 \%)$ $(\mathrm{p}=0.012) \quad(\mathrm{OR}=2.74 ; \quad(95 \%$ CI $1.210-6.186))$; and represented a significant survival disadvantage at 1 year in a Kaplan-Meier analysis (readmitted within 30 days: mean survival 348 days (95\% CI 337-359) vs not readmitted within 30 days: mean survival 361 days (95\% CI 358-363). Log rank $\mathrm{p}=0.01$ ).

The main reasons for admissions were deranged LFTs $(34 \% ; n=42)$, AKI $(22 \% ; n=27)$, and infection $(18 \% ; n=22)$.

Significant differences on multivariate analysis were found for haemoglobin at transplant $(\mathrm{HR}=0.988 \quad(95 \%$ CI 0.979 $0.996) ; \mathrm{p}=0.005)$ and creatinine at discharge $(\mathrm{HR}=1.006$ (95\% CI 1.003-1.010); $\mathrm{p}=0.001)$.

Discussion In Scotland, readmission rates following LT were lower than in previously published, American, data. Haemoglobin and creatinine were predictors of readmission.

Patients readmitted within 30 days of LT were more than twice as likely to die within 1 year.

The commonest reasons for readmission were deranged LFTs, AKI and infection.

\section{P079 APPROACH TO PRIMARY BILIARY CHOLANGITIS MANAGEMENT IN A DISTRICT GENERAL HOSPITAL}

Usama Aslam*, Mehreen Mudassar, Mohsin Munir. Ipswich Hospital/esneft, Ipswich, UK

\subsection{6/gutjnl-2021-BASL.87}

Introduction Primary biliary cholangitis (PBC) is a rare progressive immune-mediated liver disease that, if not adequately treated, may culminate in end-stage disease and need for transplantation. Both genetic and environmental influences are presumed relevant to disease initiation. PBC is most prevalent in women and those over the age of 50, but a spectrum of disease is recognised in adult patients globally; male sex, younger age at onset $(<45)$ and advanced disease at presentation are baseline predictors of poorer outcome. According to current guidelines, $\mathrm{PBC}$ is diagnosed in the presence of antimitochondrial antibodies (AMA) or specific antinuclear antibodies, and of a cholestatic biochemical profile, while biopsy is recommended only in selected cases. All patients receive ursodeoxycholic acid (UDCA) in first line; the only registered second-line therapy is obeticholic acid (OCA) for UDCA-inadequate responders

Method Retrospective review of clinical records of patients with antimitochondrial antibody positive status were reviewed by help of local immunology department. Patients involved were the ones who were AMA positive between 2015 to 2020. 26 patients were included in this review and results are summarised below

Results Out of 26 patients 11 were AMA positive only without any LFTs abnormality so no treatment was started, were planned to be followed up by yearly LFTs.

1 patient was AMA positive plus abnormal LFTs and histology consistent with PBC who presented with developed cirrhosis and variceal bleed requiring TIPPS so services were transferred to tertiary care so no record on our system.

14 patients out of 26 were AMA positive and cholestatic LFT abnormality. 6 patients out of 14 were on urso dose of $13-15 \mathrm{mg} / \mathrm{kg}$. 5 patient out of 14 had record of pruritis and fatigue documented. Only 3 patients out of 14 had DEXA scan done in last 5 years. Only 2 had developed cirrhosis who were not considered for transplant due to multiple comorbidites but were followed by as per guideline for HCC and varices.

None of these 26 patients had overlapping diagnosis of autoimmune hepatitis.

Conclusion Significant issues were identified in management of patients with PBC when it comes to correct dose of ursodeoxycholic acid and mentioning about symptoms of fatigue and pruritis. Initial management needs to be optimized before consideration of obetocholic acid for these patients

\section{P080 SOCIOECONOMIC DEPRIVATION AND ITS EFFECT ON REFERRAL FOR AND OUTCOMES FOLLOWING LIVER TRANSPLANTATION}

Thomas Manship*, Andrew Robertson, Hannah McDowell, Zara Aiken, Thomas Clouston, Kenneth Simpson. NHS Lothian, UK

\subsection{6/gutjnl-2021-BASL.88}

Introduction In Scotland lower socio-economic status is associated with a greater risk of liver-related mortality. Limited data exist on whether socio-economic status influences referral for liver transplantation (OLT) or outcomes following listing and OLT.

Methods Adult patients with chronic liver disease referred for the first time for OLT between January 2009 and December 2018 were identified. Deprivation score related to postcode (Scottish Index of Multiple Deprivation [SIMD]) was recorded. Severity of liver disease, listing decision and post listing outcomes were documented. SIMD groups were paired into quintiles $(1=$ most deprived, $5=$ least deprived). Liver related deaths in Scotland were used as an estimate of severe liver disease in the remaining population. We calculated the chance of referral for each group as follows: (number of referrals/((number of 


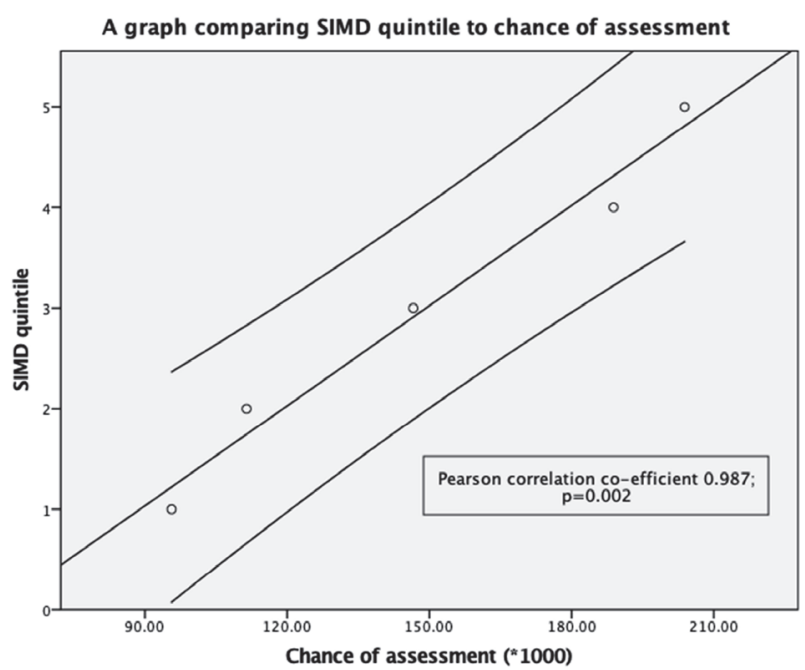

Abstract P080 Figure 1 A graph comparing SIMD quintile to chance of assessment

referrals-deaths on the list) + liver disease mortality in Scotland)

Results 1297 patients were identified. Patients from the least deprived areas of Scotland were significantly more likely to be referred for liver transplantation. Pearson Correlation co-efficient $0.987 ; p=0.002$. (See figure 1 ).

Those patients from the most deprived quintile were significantly less likely to be listed compared to the most affluent quintile. $(\mathrm{OR}=0.653 ; 95 \%$ CI $0.456-0.935 ; \mathrm{p}=0.02)$. No significant differences were found when comparing the other quintiles to the least deprived group. We did find that those from the more affluent groups did have more severe liver disease by UKELD (ANOVA $p=0.003$ ) however there was no difference in those not listed due to being 'too unwell' across the groups (Chi-squared $\mathrm{p}=0.447$ ). Number of hepatocellular carcinomas (HCC) were not significantly different across the groups (Chi-squared $\mathrm{p}=0.976$ )

Those not listed due to high risk or recidivism (Chisquared $\mathrm{p}=0.066$ ) and due to HCC outside criteria (Chisquared $\mathrm{p}=0.870$ ) were similar across the SIMD groups.

There was no difference in waiting times on the transplant list across the SIMD groups (ANOVA $\mathrm{p}=0.794$ )

We found that in those patients transplanted $(n=623)$ there was no significant difference in survival at two years when compared to the most affluent quintile. For example, when comparing the most deprived quintile (14 deaths at 2 years $(26.9 \%)$ vs 6 (11.5\%); OR $=0.600 ; 95 \%$ CI $0.222-1.621$; $\mathrm{p}=0.314)$.

Discussion Those patients from more deprived areas of Scotland are less likely to be referred or listed for liver transplantation compared to most affluent groups. However, these differences don't continue when looking at survival posttransplant.

\section{P081 DERANGED LIVER FUNCTION TESTS IN COVID POSITIVE PATIENTS REQUIRING HOSPITAL ADMISSION AT IPSWICH HOSPITAL, UK}

Usama Aslam*, Mehreen Mudassar, Mohsin Munir, Zaffar Ullah. Ipswich Hospital/esneft, Ipswich, UK

10.1136/gutjnl-2021-BASL.89
Introduction SARS-CoV-2 is a novel coronavirus that emerged in Wuhan, China in late 2019 and since become a global pandemic. Initial reports suggested a significant proportion of patients have abnormal liver blood tests. We conducted a retrospective review of clinical data to access for incidence, clinical pattern, and severity of liver blood test in patients with confirmed COVID-19.

Method Clinical records and laboratory results were obtained from 210 patients with laboratory-confirmed COVID-19 who were admitted to Ipswich Hospital, East of England, UK between March 2020 and June 2020. Only admission blood tests were reviewed and all patients who had abnormal admission liver function tests were then screened for any risk factors for liver disease. To describe the severity of liver injury, in this study, patients who had raised liver function parameters more than $5 \times$ the upper limit unit of normal (ULN) were classified as significant liver injury; patients who had raised liver function parameters 2-5 ULN were classified as moderate liver injury; and patients who had raised liver function parameters 1-2 ULN were classified as mild liver injury

Results 210 hospitalised COVID-19 positive had liver blood tests available for analysis. 44 patients (20\%) had one or more abnormal liver blood tests with raised levels of Creactive protein and procalcitonin. Abnormality in liver function test was more hepatocellular but 8 patients out of 44 had raised alkaline phosphatase only along with raised GGT but normal rest of liver enzymes. Out of these 44 patients $20(9 \%)$ required ITU admission within 24 to 48 hours of hospital admission. 15 (7\%) patients out of 44 were not ITU candidates either due to advanced age and frailty or multiple co-morbidities. 9 patients out 44 were managed on ward as were not too unwell to require ITU admission. All patient reviewed in our data had mild to moderate liver injury with none having severe injury. Factors identified in patient requiring ITU admission was male gender, age greater than 60 years and multiple comorbidities.

Conclusion Twenty percent of patients admitted to the hospital with SARS-CoV-2 infection had an abnormal liver function which was found to be associated with raised levels of inflammatory markers. Its important to aggressively treat patients with COVID-19 infection and deranged LFTS as there is a risk involved that these patients might require ITU. Its important to perform liver screen blood tests along with imaging of liver

\section{P082 VALIDATION OF THE BAVENO VI CRITERIA: IDENTIFYING LOW RISK CIRRHOTIC PATIENTS NOT REQUIRING ENDOSCOPIC SURVEILLANCE IN A TEACHING HOSPITAL}

Daniyal Baig*, John Ross Briggs, Cyril Sieberhagen. Aintree University Hospitals NHS Foundation Trust, UK

\subsection{6/gutjnl-2021-BASL.90}

Introduction In cirrhotic patients, oesophageal varices are one of the major causes of death (6-week mortality is between $17 \%-28 \%)$ and $5-11 \%$ of all cases of upper gastrointestinal bleeding (UGIB).

Patients with cirrhosis undergo variceal surveillance with oesophago-gastroduodenoscopy (OGD) to reduce the risk of oesophageal variceal bleeding. Patients with compensated 